\title{
Avaliação da infiltração marginal de restaurações de resina composta com diferentes sistemas adesivos e tempos de armazenagem
}

\section{Marginal leakage evaluation of composite restorations with different adhesive systems and storage times}

\author{
Marcos Beleza Galvão VIEIRA \\ Doutorando - Programa de Pós-Graduação em Odontologia - Faculdade de Odontologia de Pernambuco - Universidade \\ de Pernambuco - UPE - Camaragibe - PE.
}

\section{Maria das Neves CORREIA}

Professor Adjunto - Área de Dentística - Faculdade de Odontologia de Pernambuco - Universidade de Pernambuco UPE - Camaragibe - PE.

\section{Rodivan Brás da SILVA}

Professor Adjunto - Área de Dentística - Faculdade de Odontologia de Pernambuco - Universidade de Pernambuco UPE - Camaragibe - PE.

\section{Mário Alexandre Coelho SINHORETI}

Professor Titular - Área de Materiais Dentários - Faculdade de Odontologia de Piracicaba - Universidade Estadual de Campinas - UNICAMP - Piracicaba - SP

\begin{abstract}
Resumo
O objetivo deste trabalho foi avaliar in vitro a infiltração marginal de restaurações de compósito utilizando diferentes sistemas adesivos: Adper Single Bond 2 (3M/ESPE), Scotchbond Multi-uso (3M-ESPE), Clearfil SE Bond (Kuraray) e Adper Prompt L-Pop (3M-ESPE). Foram utilizados oitenta dentes bovinos, que foram separados aleatoriamente em quatro grupos, de acordo com o sistema adesivo utilizado. Os dentes tiveram cavidades circulares (2mm diâmetro X $2 \mathrm{~mm}$ profundidade) preparadas na porção radicular utilizando pontas diamantadas. Os dentes foram restaurados em único incremento de compósito e armazenados por 24 horas ou 3 meses. Após os períodos de armazenagem, os dentes de cada grupo $(n=10)$ foram vedados com duas camadas de esmalte para unha e submetidos a 500 ciclos térmicos $\left(5-55^{\circ} \mathrm{C}\right)$, imersos no corante azul de metileno a $2 \%$. Após, as coroas e terço apical das raízes foram seccionadas e descartadas, ficando apenas a porção do terço cervical e médio. Essa porção que continha a restauração, foi seccionada no sentido vestíbulo-lingual para a avaliação da infiltração marginal através de lupa estereoscópica com 40X de aumento. O critério utilizado para a avaliação da infiltração foi o seguinte: houve infiltração do corante e não houve infiltração do corante. Os resultados foram submetidos ao teste de Fisher e ao teste de McNemar. Não houve diferença significativa entre os sistemas adesivos, seja no período de armazenagem de 24 horas ou de 3 meses, com exceção do Adper Single Bond 2, onde no período de 24 horas houve estatisticamente maior infiltração do que em 3 meses.
\end{abstract}

\section{UNITERMOS}

Resinas compostas; infiltração dentária; restauração dentária permanente. 


\section{INTRODUÇÃO}

O maior desafio no caminho evolutivo dos sistemas adesivos sempre foi conseguir união à dentina, já que é um substrato complexo e apresenta, além do conteúdo inorgânco, matéria orgânica, umidade e maior proximidade da polpa ${ }^{13}$. Outra característica da dentina é a presença da smear layer que se forma na superfície após a instrumentação, a qual oblitera a entrada dos túbulos dentinários e diminui a permeabilidade dentinária em cerca de $86 \%{ }^{17,20}$. Além disso, essa camada reduz a energia livre superficial da dentina, podendo interferir no processo adesivo ${ }^{3}$. Se a união não for efetiva, poderá ocorrer o rompimento da união e, conseqüentemente, a infiltração de microorganismos, fluidos e moléculas na interface dente-restauração ${ }^{1,6}$, ${ }^{13}$. Assim, a infiltração marginal pode ser considerada um dos fatores que mais influenciam a longevidade das restaurações, podendo promover a perda precoce do procedimento restaurador pelo aparecimento de cárie recorrente, sensibilidade pós-operatória ou danos pulpares ${ }^{9,19}$.

Outro aspecto de grande importância em relação à microinfiltração é o problema da contração de polimerização das resinas compostas, podendo gerar forças capazes de romper a união do material à parede cavitária, resultando na formação de fendas ${ }^{7}$.

As primeiras gerações dos sistemas adesivos dentinários tinham como mecanismo de ação a união iônica com a hidroxiapatita ou uma união covalente (união de hidrogênio) com o colágeno. Esses materiais eram hidrófobos e tendem a atacar de forma limitada a "smear layer", gerando resistência de união muito baixa $^{12}$. E assim surgiram as gerações mais recentes onde a dentina passou a ser condicionada por agentes ácidos, removendo a smear layer. Os sistemas adesivos dessa geração contêm um condicionador ácido o qual é sempre lavado após o condicionamento, uma solução de monômeros hidrófilicos chamados "primers", geralmente dissolvidos em álcool (etanol) e/ou acetona, e uma resina fluida, com ou sem carga ${ }^{22}$.

No entanto, essa técnica de condicionamento ácido total ("total-etch"), promove um ataque ácido da dentina sem uniformidade, o que poderá gerar dificuldade de penetração dos monômeros nos espaços ao redor das fibras colágenas e da dentina peritubular. Isso poderá levar à formação de áreas susceptíveis à infiltração pela deficiência na penetração do "primer" e do adesivo ${ }^{21}$.

Nesse sentido, os sistemas adesivos autocondicionantes tentam resolver essas dificuldades, pois eliminam o problema da infiltração incompleta dentro da região desmineralizada pelo sistema adesivo $^{4}$. A vantagem desse sistema, é que o condicionamento ácido e penetração do adesivo são feitos simultaneamente ${ }^{28}$. Teoricamente, nesses sistemas adesivos, o "primer" dissolve e incorpora a "smear layer" dentro da mistura, além de desmineralizar a dentina e encapsular as fibras colágenas e os cristais de hidroxiapatita. Simultaneamente, os monômeros penetram nessa região, os quais podem ser polimerizados "in situ"4.

A água é um componente essencial nos sistemas adesivos autocondicionantes, pois permite que os monômeros ácidos sejam efetivamente ionizados, para então desmineralizar o substrato dental ${ }^{28}$. Esses "primers" combinam dois passos (condicionamento ácido e "priming") dentro de um único passo. As superfícies condicionadas são subseqüentemente cobertas com camadas de adesivos hidrofóbicos que são fotopolimerizados ${ }^{29}$. O último avanço nessa tecnologia adesiva são os sistemas adesivos autocondicionantes todos-em-um ("all-in-one) que simultaneamente condicionam o dente e penetram no substrato dental em uma única soluçãa ${ }^{28}$. Esses adesivos são extremamente hidrófilos, contendo altas concentrações iônicas e monômeros hidrófilos.

Os sistemas adesivos convencionais são altamente sensíveis à secagem da cavidade, além de produzirem uma camada de adesivo não uniforme. Já, os sistemas adesivos autocondicionantes, principalmente os de passo único, são extremamente hidrófilos, aumentando seu potencial de degradação $0^{4,10}$. Todos os sistemas, porém, são membranas permeáveis e degradáveis. Então é relevante pesquisar se o tempo de armazenamento influenciará no selamento marginal, uma vez que os sistemas a serem pesquisados possuem características diferentes.

Desse modo, o objetivo desse trabalho foi comparar diferentes sistemas adesivos e verificar a influência do tempo de armazenagem ( 24 horas e três meses) sobre a infiltração marginal em restaurações de resina composta.

\section{MateriaIS e MÉtodo}

Foram utilizados oitenta dentes incisivos centrais bovinos $(\mathrm{N}=80)$. Na superfície proximal mais plana de cada raiz foi confeccionado um preparo cavitário circular com $2 \mathrm{~mm}$ de diâmetro por $2 \mathrm{~mm}$ de profundidade. Após, as cavidades foram restauradas com o compósito Z250 (3M/ESPE), na cor A3, usando 
os seguintes sistemas adesivos: Adper Single Bond2 (3M/ESPE), Scotchbond Multi-Purpose (3M/ESPE), Clearfil SE Bond (Kuraray) ou Adper Prompt L-Pop (3M-ESPE). Os corpos-de-prova foram separados em quatro grupos, de acordo com o tipo de sistema adesivo e, subdivididos em dois grupos, de acordo com o tempo de armazenamento de 24 horas ou três meses (Quadro 1).

Quadro1 - Divisão dos grupos experimentais $(n=10)$

\begin{tabular}{l|l|l|l|l}
\hline Grupo & Subgrupo & Sistema adesivo & N & Tempo de armazenamento \\
\hline G1 & A & Adper Single Bond 2 & 10 & 24 horas \\
G1 & B & Adper Single Bond 2 & 10 & 3 meses \\
G2 & A & A.Scotchbond Multi-Purpose & 10 & 24 horas \\
G2 & B & A.Scotchbond Multi-Purpose & 10 & 3 meses \\
G3 & A & Clearfil SE bond & 10 & 24 horas \\
G3 & B & Clearfil SE bond & 10 & 3 meses \\
G4 & A & Adper Prompt L- Pop & 10 & 24 horas \\
G4 & B & Adper Prompt L- Pop & 10 & 3 meses \\
\hline
\end{tabular}

Todos os procedimentos restauradores foram feitos seguindo-se as recomendações de cada fabricante. Decorrida esta etapa, os corpos-de-prova foram armazenados em água destilada à $37^{\circ} \mathrm{C}$ por 24 horas ou 3 meses. Após o tempo de armazenamento, os corpos-de-prova de todos os grupos foram submetidos à ciclagem térmica em banhos com água à temperatura de $5-55^{\circ} \mathrm{C} \pm 2^{\circ} \mathrm{C}$, por $15 \mathrm{~s}$, num total de 500 ciclos. No passo seguinte, os forames apicais foram vedados com resina composta, complementado com a aplicação de cola de presa rápida. Duas camadas de esmalte para unhas (Revlon) foram usadas para cobrir toda superfície dos corpos-de-prova, exceto a região da restauração e $1 \mathrm{~mm}$ aquém de suas margens. Em seguida, foram imersos em solução de azul de metileno a 2\% (pH ajustado em 7), por 2 horas. Após foram lavados em água corrente por 5 minutos para remoção do excesso de corante, secos com papel absorvente e, seccionados no sentido longitudinal, passando pelo centro da restauração. As duas partes obtidas foram então submetidas à avaliação numa lupa estereoscópica Carl Zeiss (40x de aumento), por três examinadores previamente calibrados, que observavam os seguintes escores: 0 - não houve penetração do corante na interface dente restauração e; 1 - houve penetração do corante na interface denterestauração. Os dados foram tratados estatisticamente através do teste exato de Fisher e McNemar ao nível de significância de $5 \%$.

\section{Resultados}

A Tabela 1 mostra a percentagem de infiltração nos períodos de armazenagem de 24 horas e três meses, para os diferentes sistemas adesivos. O resultado do teste exato de Fisher mostrou que não houve diferença estatisticamente significativa nas percentagens de infiltração marginal entre os diferentes sistemas adesivos, tanto no período de 24 horas, como no período de três meses de armazenamento. Para a comparação dos períodos de armazenamento, dentro de cada sistema adesivo, foi utilizado o teste de McNemar. Foi observado que houve diferença estatística na percentagem de infiltração apenas no sistema adesivo Adper Single Bond 2, entre os períodos de 24 horas (maior infiltração) e três meses de armazenagem. 
AVALIAÇÃO DA INFILTRAÇÃO MARGINAL DE RESTAURAÇÕES DE RESINA COMPOSTA COM DIFERENTES SISTEMAS ADESIVOS E TEMPOS DE ARMAZENAGEM

\section{Tabela 1 - Freqüência de infiltração marginal ocorrida nos períodos de armazenagem de 24 horas e de três meses, para os diferentes sistemas adesivos}

\begin{tabular}{lll}
\hline & \multicolumn{2}{l}{ Tempo de armazenagem } \\
\cline { 2 - 3 } Sistema adesivo & 24 horas & 3 meses \\
\hline Adper Single Bond 2 & $7(70,0 \%) \mathrm{a}^{*}$ & $1(10,0 \%) \mathrm{b}^{\star \star}$ \\
Scotchbond Multi-uso & $9(90,0 \%) \mathrm{a}^{*}$ & $6(60,0 \%) \mathrm{a}^{\star *}$ \\
Clearfil SE bond & $5(50,0 \%) \mathrm{a}^{*}$ & $6(60,0 \%) \mathrm{a}^{\star \star}$ \\
Adper Prompt L-Pop & $6(60,0 \%) \mathrm{a}^{*}$ & $4(40,0 \%) \mathrm{a}^{\star *}$ \\
\hline
\end{tabular}

As letras foram utilizadas para indicar comparações de percentagens apenas entre colunas ( 24 horas versus 3 meses); * compara as percentagens das linhas às 24 horas de armazenagem; ** compara as percentagens das linhas aos 3 meses de armazenagem.

\section{DIscussão}

Neste estudo foram utilizados sistemas adesivos com diferentes modos de aplicação e atuação no substrato dental, bem como, tempo de armazenagem distintos. Os sistemas adesivos que apresentaram os melhores resultados em ordem decrescente após 24 horas de armazenagem, mesmo não havendo diferenças significantes foram: Clearfil SE Bond, Adper Prompt L- Pop, Adper Single Bond 2 e Scotchbond MultiPurpose. Os sistemas Clearfil SE Bond e o Adper Prompt L-Pop são sistemas adesivos autocondicionantes e, portanto, dispensam o uso de ataque ácido. Estes sistemas removem parcialmente ou totalmente a smear layer. Nesta situação ele pode guardar o material incorporado na camada de resina, enquanto penetra no substrato, criando a camada híbrida que contém a smear layer original ${ }^{8}$. Outro aspecto importante desses sistemas é que os sistemas autocondicionantes podem ser classificados segundo o seu $\mathrm{pH}$, em: médio, intermediário e agressivo. Essa característica modifica sua capacidade de penetrar na smear layer e na superfície da dentina desmineralizada. Os sistemas adesivos mais agressivos solubilizam completamente a smear layer e formam muitas vezes, uma camada híbrida com espessura aproximada daqueles sistemas adesivos que usam condicionamento ácido prévio ${ }^{28}$.

Assim, no período de armazenagem de 24 horas, apesar de não ter havido diferença estatística, o sistema adesivo autocondicionate Clearfil SE Bond apresentou menor frequiência de infiltração marginal do que o sistema adesivo autocondicionante Adper Prompt LPop. A provável causa dessa maior freqüência, talvez esteja no fato de que, devido ao ataque brando do primer acídico, este retém a smear layer da dentina hibridizada dentro dos túbulos dentinários, o que reduz o fluido externo, resultando em um selamento superior $^{11}$. Nesse mesmo raciocínio, o sistema adesivo que apresentou maior frequiência de infiltração marginal no período de 24 horas de armazenagem foi o Scotchbond Mult-Uso. Uma explicação para isso talvez esteja no primer desse sistema adesivo que contém água na composição, o que tenderia a dar melhores resultados somente quando a dentina estivesse seca ("dry bonding technique") ${ }^{14}$. Uma outra questão que se pode levantar para o mal desempenho do sistema adesivo Scotchbond Multi-Uso está no fato do fabricante recomendar uma única aplicação tanto do primer, quanto do agente de união. Walshaw \& $\mathrm{McComb}^{30}$ (1996) e Choi et al. ${ }^{5}$ (2000) estudaram as mudanças que ocorrem na tensão de contração de polimerização quando do uso de várias camadas desse sistema adesivo na redução da microinfiltração em cavidades de classe $\mathrm{V}$ e demonstraram que a tensão de contração de polimerização diminuiu significantemente quando a espessura do sistema adesivo foi aumentada, reduzindo também o nível de microinfiltração.

Quando os mesmos sistemas foram avaliados no período de armazenagem de três meses, também não houve diferença estatisticamente significante, porém os melhores resultados apresentados para os diferentes sistemas testados, do melhor para o pior desempenho, foram os seguintes: Adper Single Bond 2, Adper Prompt L- Pop, Scotchbond Multi-Uso, Clearfil SE Bond. Os dois últimos apresentaram os mesmos percentuais de infiltração marginal, como também foram os que apresentaram os piores resultados com relação à infiltração marginal. 
Comparando-se cada sistema correspondente entre os dois períodos de armazenagem (24 horas versus três meses), apenas o sistema Adper Single Bond 2, apresentou resultados estatisticamente significante e superiores no período de três meses em relação ao período de 24 horas. Essa melhora, provavelmente, se deve ao fato que na composição desses sistemas existem partículas esféricas de sílica com diâmetro de 5 nanômetros. Esta carga representa $10 \%$ do peso total do adesivo e são tratadas com silano, incorporando-se à matriz resinosa do adesivo através de um processo que evita a sua aglomeração $0^{23}$. Talvez esse tipo de camada de adesivo formada possa absorver as tensões da contração de polimerização ao longo do tempo com maior eficiência do que os demais sistemas adesivos. A não observância de diferenças estatisticamente significantes nos demais sistemas provavelmente esteja no fato do período de armazenagem ser de apenas três meses, estando de acordo com o trabalho de Sadek et al. ${ }^{25}$ (2003) que demonstrou que o tempo de armazenagem de três meses não influenciou no comportamento dos sistemas adesivos, não permitindo a degradação hidrolítica na interface de união ${ }^{15}$.

No entanto, nenhum sistema adesivo eliminou totalmente a infiltração marginal. Suppa et al. ${ }^{27}$ (2005) já tinham verificado que nenhum sistema adesivo, quer seja convencional ou autocondicionante, foi capaz de envolver todas as fibras colágenas, favorencendo a degradação hidrolítica. O trabalho desses autores vem corroborar com o nosso, que embora utilizando metodologias diferentes, têm em comum a determinação do selamento marginal de restaurações em compósito.

A metodologia de infiltração marginal utilizando corantes orgânicos ou inorgânicos tem sido amplamente utilizada, devido à rapidez para obtenção dos resultados e facilidade de execução ${ }^{2}$. Porém, seus resultados podem ser parcialmente ou totalmente influenciados pelo tipo de metodologia empregada, o que limita a comparação entre os resultados obtidos por diferentes estudos ${ }^{2}$. Raskin et al. ${ }^{24}$ (2001), afirmam que a metodologia é ampla, podendo variar quanto à amostra, preparo cavitário, emprego de termociclagem, ciclagem mecânica, tempo de imersão no corante, quantidade de corte, calibração dos avaliadores e também pelo tipo de substrato dentário utilizado.

Em relação a esse último fator, deveria-se utilizar preferencialmente dentes humanos para esse tipo de teste. No entanto, a obtenção de dentes humanos tem se tornado cada vez mais difícil, devido principalmente ao avanço da Odontologia preventiva. Com isso, tornou-se necessária a busca por um substrato alternativo ao dente humano. Devido ao fato de que os dentes das diferentes espécies de mamíferos são similares, tanto morfologicamente quanto histologicamente, muitas pesquisas foram desenvolvidas utilizando-se dentes bovinos, caninos, suínos, primatas e outros ${ }^{18}$. Em relação aos dentes bovinos, Schilke et al. ${ }^{26}$ (2005), afirmaram que a morfologia dos túbulos dentinários, tanto em número por $\mathrm{mm}^{2}$ quanto em diâmetro, é similar quando comparadas à dentina coronal dos incisivos decíduos humanos, dos molares permanentes humanos e dos incisivos centrais bovinos. Assim, os dentes bovinos são uma alternativa viável aos dentes humanos em testes laboratoriais, porém seria aconselhável ter um pouco de cautela e critério durante sua utilização ${ }^{16}$.

Caberia também avaliar as outras variáveis que atuam diretamente na estabilidade de união entre o compósito e o substrato dental, como a ciclagem térmica mecânica e maior número de ciclos térmicos. Talvez sob a ação dessas variáveis, o comportamento desses sistemas de união poderia ser diferente. Mas, sempre é válido ressaltar que para o entendimento do fenômeno "efeito de armazenagem e tipo de sistema adesivo", esse estudo mostrou parcialmente a influência dessas variáveis. Para um completo entendimento do assunto, vários estudos seriam necessários.

\section{Conclusão}

Diante dos resultados, pode-se concluir que:

- Nenhum sistema adesivo foi capaz de eliminar totalmente a infiltração marginal;

- Não houve diferença estatística entre os sistemas adesivos, tanto no período de 24 horas como no período de 3 meses de armazenagem;

- O sistema adesivo Adper Single Bond 2 mostrou maior infiltração marginal no período de 24 horas quando comparado ao período de 3 meses de armazenagem; e

- Para os demais sistemas adesivos estudados não foram observadas diferenças na infiltração marginal entre os períodos de armazenagem. 


\section{Abstract}

The aim of this study was to evaluate the in vitro marginal leakage of resin composite restorations when different adhesive systems are applied: Adper Single Bond 2 (3M/ESPE), Scotchbond Multi-uso (3M-ESPE), Clearfil SE Bond (Kuraray) and Adper Prompt L-Pop (3M-ESPE). Eighty bovine incisors were distributed into four groups, according to the adhesive treatment. Circular cavities $(2 \mathrm{~mm} \emptyset ; 2 \mathrm{~mm}$ depth) were prepared on the root portions with diamond burs. The teeth were restored with resin composite, by single increment, and stored during 24 hours or three months (eight groups; $n=10$ ). Therefore, two layers of nail varnish were applied. The samples were submitted to 500 thermo-cycles $\left(5-55^{\circ} \mathrm{C}\right)$ while immersed in blue ink $(2 \%$ methylene blue). Sections were made in the cementum-enamel junction and apical regions. Just the restored part was cross-sectioned in order to evaluate the marginal infiltration through stereomicroscope light (40X). The following criteria were used: with or without ink penetration. The statistical comparisons were performed by use of Fisher and McNemar tests. There were no statistical differences among the adhesive systems, regardless of the storage time. The only exception was the Adper Single Bond 2, where the 24 hours evaluation showed higher leakage than the three months one.

\section{UNITERMS}

Composite resins; dental leakage; dental restoration permanent.

\section{REFERÊNCIAS}

1. Alani AH, Toh CG. Detection of microleakage around dental restorations: a review. Oper Dent. 1977 July/Aug.;22 (4):173-85.

2. Amarante de Camargo DA, Sinhoreti MA, Correr-Sobrinho L, de Sousa Neto MD, Consani S. Influence of the methodology and evaluation criteria on determining microleakage in dentin-restorative interfaces. Clin Oral Investig. 2006 Dec;10(4):317-23.

3. Bertolotti RL. Conditioning of the dentin substrate. Oper Dent. 1992;Suppl 5:131-6.

4. Carvalho RM, Pegoraro TA, Tay FR, Pegoraro LF, Silva NR, Pashley DH. Adhesive permeability affects coupling of resin cements that utilise self-etching primers to dentine. J Dent. 2004 Jan;32(1):55-65.

5. Choi KK, Condon JR, Ferracane JL. The effects of adhesive thickness on polymerization contraction stress of composite. J Dent Res. 2000 Mar;79(3):812-7.

6. Cox CF, Keall CL, Keall HJ, Ostro E, Bergenholtz G. Biocompatibility of surface-sealed dental materials against exposed pulps. J. Prosthet Dent. 1987 Jan;57 (1):1-8

7. Davidson CL, de Gee AJ. Relaxation of polymerization contraction stresses by flow in dental composites. J Dent Res. 1984 Feb;63(2):146-8.

8. Ferrari M, Tay FR. Technique sensitivity in bonding to vital, acid-etched dentin. Oper Dent. 2003 Jan-Feb;28(1):3-8.

9. Going RE. Microleakage around dental restorations: a summarizing review. J Am Dent Assoc. 1972 Jun;84(6):1349-57.

10. Hashimoto M, Ohno H, Sano H, Kaga M, Oguchi H. In vitro degradation of resin-dentin bonds analyzed by microtensile bond test, scanning and transmission electron microscopy. Biomaterials. 2003 Sep;24(21):3795-803.

11. Hashimoto M, Ito S, Tay FR, Svizero NR, Sano H, Kaga M, et al. Fluid movement across the resin-dentin interface during and after bonding. J Dent Res. 2004 Nov;83(11):843-8.

12. Heymann HO, Bayne SC. Current concepts in dentin bonding: focusing on dentinal adhesion factors. J Am Dent Assoc. 1993 May;124(5):26-36.

13. Kidd EA. Microleakage a review. J Dent. 1976 Sep;4(5):199-206.
14. Konno AN, Sinhoreti MA, Consani S, Correr Sobrinho L, Consani RL. Storage effect on the shear bond strength of adhesive systems. Braz Dent J. 2003;14(1):42-7.

15. Li HP, Burrow MF, Tyas MJ. The effect of long-term storage on nanoleakage. Oper Dent. 2001 Nov-Dec;26(6):609-16.

16. Lopes MB, Sinhoreti MA, Correr Sobrinho L, Consani S. Comparative study of the dental substrate used in shear bond strength tests. Pesqui Odontol Bras. 2003 Apr-Jun;17(2):171-5.

17. Matloff IR, Jensen JR, Singer L, Tabibi A. A comparison of methods used in root canal seability studies. Oral Surg Oral Med Oral Pathol. $1982 \mathrm{Feb} ; 53(2): 203-8$.

18. Nakamichi I, Iwaku M, Fusayama T. Bovine teeth as possible substitutes in the adhesion test. J Dent Res. 1983 Oct; 62(10):1076-81.

19. Pashley DH. Clinical considerations of microleakage. J Endod. 1990 Feb;16(2):70-7.

20. Pashley DH, Livingston MJ, Whitford GM. The effect of molecular size on reflection coefficients in human dentine. Arch Oral Biol. 1979;24(6):455-60.

21. Pashley DH, Carvalho RM. Dentine permeability and dentine adhesion. J Dent. 1997 Sep;25(5):355-72.

22. Perdigao J, Geraldeli S, Hodges JS. Total-etch versus self-etch adhesive: effect on postoperative sensitivity. J Am Dent Assoc. 2003 Dec;134(12):1621-9.

23. Perfil técnico do produto Sistema adesivo Adper Single Bond 2: Saint Paul: 3M/ESPE, 2004. Acesso em: 24 de nov. 2005.Disponível em: http://www.3m.com/intl/br/espe/catalogos /AdperSingleBond2perfilPTrev270504.pdf.

24. Raskin A, D'Hoore W, Gonthier S, Degrange M, Dejou J.. Reliability of in vitro microleakage testes: a literature review. J Adhes Dent. 2001; 3(4):295-308

25. Sadek FT, Moura SK, Ballester RY, Muench A, Cardoso PE. The effect of long-term storage on the microleakage of composite resin restorations: qualitative and quantitative evaluation. Pesqui Odontol Bras. 2003 Jul-Sep;17(3):261-6. 
AVALIAÇÃO DA INFILTRAÇÃO MARGINAL DE RESTAURAÇÕES DE RESINA COMPOSTA COM DIFERENTES SISTEMAS ADESIVOS E TEMPOS DE ARMAZENAGEM

26. Schilke R, Lisson JA, Bauss O, Geurtsen W. Comparison of the number and diameter of dentinal tubules in human and bovine dentine by scanning electron microscopic investigation. Arch Oral Biol. 2000 May;45(5):355-61.

27. Suppa P, Breschi L, Ruggeri A, Mazzotti G, Prati C, Chersoni S, et al. Nanoleakage within the hybrid layer: a correlative FEISEM/ TEM investigation. J Biomed Mater Res B Appl Biomater. 2005 Apr;73(1):7-14.

28. Tay FR, Pashley DH. Aggressiveness of contemporary self-etching systems. I: Depth of penetration beyond dentin smear layers. Dent Mater. 2001 Jul; 17(4):296-308.

29. Tay FR, Frankenberger R, Krejci I, Bouillaguet S, Pashley DH, Carvalho RM, et al. Single-bottle adhesives behave as permeable membranes after polymerization. I. In vivo evidence. J Dent. 2004 Nov;32(8):611-21.
30. Walshaw PR, McComb D. Clinical considerations for optimal dentinal bonding. Quintessence Int. 1996 Sep;27(9):619-25.

Recebido em: 26/05/07 Aprovado em: 31/07/07

Autor para correspondência: Mário Alexandre Coelho Sinhoreti e-mail: sinhoret@fop.unicamp.br Av. Limeira, 901, Bairro Areião 13414-903, Piracicaba - SP Fone: (19) 2106-5374 Fax: (19) 2106-5218 